\title{
DEMANDA Y OFERTA DE EDUCACIÓN SUPERIOR: INTEGRACIÓN TOTAL DE LA CADENA DE VALOR Y LAS CADENAS DE SUMINISTRO
}

\author{
Cesar H. Ortega Jimenez, Universidad Nacional Autónoma de Honduras, \\ Instituto de Investigaciones Económicas y Sociales (IIESUNAH), \\ Ciudad universitaria edifico c2 primer piso. Tel./Fax: 504-22348973 \\ Correo electrónico: cortega@iies-unah.org \\ Ignacio Eguía Salinas, Universidad de Sevilla, E.T.S de ingeniería, \\ Organización Industrial y Gestión de Empresas, \\ Ave. Camino de los Descubrimientos, S/N, Sevilla 41092
}

\section{RESUMEN}

Para la demanda y oferta de educación superior (ES), se propone un modelo sistémico que integra una cadena de valor y dos de suministro de ES de Honduras, que incluye los insumos/entradas de ES (ej. graduados de educación media), el proceso de ES, las salidas/resultados de la ES. Para ello, se toman todos los actores de lo que podría ser considerado como un sistema de valor y de suministro de ES (proveedores de insumos, ofertantes/universidades y demandantes/ beneficiarios de resultados) para entender como sus interrelaciones contribuye al éxito de las operaciones de las tres funciones fundamentales de ES: docencia, investigación y vinculación con la sociedad. Así pues, la perspectiva holística considera tanto a una cadena de suministro de docencia (transferencia de conocimiento a estudiantes para convertirse en profesionales como parte del capital intelectual de ES), como a una cadena de suministro de investigación/proyecto (producción de conocimiento), así como una cadena de valor de la vinculación con la sociedad, que realimente el valor percibido de los resultados de ES (profesionales e investigación) entregados a sus demandantes. Así pues, dicha integración muestra no sólo el valor añadido por el proceso de la ES a ambos suministros (docencia e investigación), sino también la productividad de ambos, mejorando continuamente el flujo bidireccional ES-sociedad (Oferta y Demanda de ES). Todo ello como fundamento para investigaciones futuras, en un proyecto de ES en marcha, que permitan la articulación eficiente y eficaz entre proveedor de insumos, ofertante de ES y demandante de ES, guiada por la demanda (valor suplido al demandante) desde un modelo de un sistema de valor y de 
suministro con integración total (una ES articulada no sólo hacia adelante por la demanda, sino también hacia atrás por la oferta).

Palabras clave: Educación superior. Cadena de suministro, Cadena de valor, Demanda, Oferta, Profesional, Investigación, Vinculación con sociedad.

\title{
DEMAND AND SUPPLY OF HIGHER EDU- CATION: TOTAL INTEGRATION VALUE CHAIN AND SUPPLY CHAINS
}

\author{
Cesar H. Ortega Jiménez, Universidad Nacional Autónoma de Honduras, \\ Instituto de Investigaciones Económicas y Sociales (IIESUNAH), \\ Ciudad universitaria edifico c2 primer piso. Tel./Fax: 504-22348973 \\ Correo electrónico: cortega@iies-unah.org \\ Ignacio Eguía Salinas, Universidad de Sevilla, E.T.S de ingeniería, \\ Organización Industrial y Gestión de Empresas, \\ Ave. Camino de los Descubrimientos, S/N, Sevilla 41092
}

\section{ABSTRACT}

For the higher education (HE) supply and demand, this paper proposes a systemic model that integrates $\mathrm{HE}$ value and supply chains in Honduras, which includes HE inputs (e.g. high school graduates), HE process, HE outputs. Thus, this paper takes all players from what may be considered as a $\mathrm{HE}$ value and supply system (i.e. HE input providers, universities, and HE output demanders seen as HE users/beneficiaries/receivers) in order to understand how their interrelationship contributes to the success of operations of the three fundamental HE functions: teaching, research and society links. Thus, the holistic view considers both a teaching supply chain (i.e. knowledge transfer to students to become professionals as part of HE intellectual capital) and a research/project supply chain (i.e. knowledge production), along with a value chain made up of society links that feeds back the value perceived of HE results (professionals and research) from society and other demanders. Hence, this integration shows not only the adding value of HE processes to both supplies (i.e. teaching and research) but also the productivity of both, thus continually improving a bidirectional HE-society flow. This will be used as a cornerstone for future research on an ongoing project that allows for an efficient and effective interrelationship among HE input providers, HE suppliers, and HE demanders, guided by demand (value supplied to demanders) from a model of value and supply system with total integration (a HE integrated not only forward by demand, but also backward by supply).

Keywords: Higher education, Supply chain, Value chain, Demand, Supply, Professional, Research, Society links. 


\section{INTRODUCCIÓN}

Se puede decir que existe un incremento en la investigación internacional sobre temas relacionados a lo que podría ser considerado como un sistema de valor y de suministro de educación superior (ES), tales como la cadena de valor, la cadena de suministro, la oferta y demanda de profesionales e investigación de ES, empleabilidad y tasa de retorno de profesionales de ES, aplicabilidad y tasa de retorno de investigaciones ${ }^{1}$, el grado de reacción a la demanda de profesionales e investigaciones de ES, la articulación ES-educación media (EM)) y la articulación ES-empleadores, entre otros (Sison y Pablo, 2000; Van der Merwe y Cronje, 2004; Watton y Truscott, 2006; Makkar et al., 2008; CFE, 2009; Froeschle, 2010; Habib, 2010; Pathak y Pathak, 2010). Por otro lado, aunque no existen investigaciones a nivel nacional, el involucramiento entre demandantes de la ES (ej. empleadores) y ofertantes de ES ha aumentado, especialmente con ciertas instituciones de educación superior (IES's), con ciertas áreas del conocimiento, y con ciertas disciplinas y grados académicos. Ello se fomenta mayormente por la nueva estrategia, parte de la reforma universitaria de la Universidad Nacional Autónoma de Honduras (UNAH), de evaluar el desarrollo potencial de aprendizajes más flexibles y vocacionales, por medio del impulso de modelos de sistemas de valor y de suministro, que integren y optimicen operaciones intra e interinstitucionalmente, satisfaciendo a todos los involucrados en el proceso de ES (proveedores de insumos, demandantes de ES, etc.).

La cadena de valor es la combinación de actividades, operando en una organización, que actuando juntas añaden valor a los demandantes/beneficiarios, por los resultados o salidas de dichas actividades (Porter, 1985). Para enfocar dicho concepto a la educación se deben considerar tres puntos en relación al valor: 1) es una experiencia subjetiva dependiente del contexto; 2) ocurre cuando las necesidades se satisfacen por la provisión de profesionales e investigaciones/ proyectos de ES (productos, recursos, procesos y servicios de la docencia e investigación); y 3) es una experiencia que fluye desde el demandante (receptor), a pesar de que la provisión fluye desde la institución fuente de recursos. Por tanto, se debe considerar también la cadena de suministro, que consiste generalmente de uno o varios proveedores de insumos, que trasladan dichos insumos a uno o varios ofertantes (ej. productores de bienes y/o servicios) para procesar los insumos y luego entregar los resultados a uno varios demandantes que los usan o se benefician de ellos (flujo de oferta de bienes y servicios, de la fuente de suministro al demandante). Opuesta al flujo de oferta, la cadena de valor tiene un flujo de demanda (conocido también como cadena de demanda) de valor (paralelo junto con flujos de peticiones/solicitudes/entradas y dinero) desde el beneficiario/demandante hasta el ofertante, en forma de demanda: el demandante es la fuente de estimación del valor (Walters y Rainbird, 2004; 
Feller et al., 2006). Además, debe existir un flujo de información bidireccional entre todos los actores en la cadena. Finalmente, la interconexión de la cadena de valor (en su vinculación con la sociedad) con las dos cadenas de suministro (docencia e investigación) involucradas en la ES puede ser considerada como un sistema de valor y de suministro de ES (Porter, 1985).

Así pues, la educación superior nacional puede ser modelada usando los conceptos de la cadena de valor y la de suministro en su búsqueda de mejoras en las operaciones, en la satisfacción de demandantes, en resultados de calidad y en los procesos de externalización (subcontratación), entre otros. Además, el estudio de ambos tipos de cadenas puede ser una necesidad imperante por la importancia actual en los servicios de Nueva Tecnología de la Información y Comunicaciones (NTIC), por la tendencia hacia la globalización en general (y en especial de oferta y producción de bienes y servicios), por las presiones competitivas y el enfoque en la innovación como parte de la estrategia, por la tendencia en los estudios de gestión (incluida la evolución en modelos de gestión para iniciativas que extiendan los servicios de ES hacia la sociedad), y por el demandante mismo de las cadenas y la complejidad del entorno (Stevenson, 2002; Feller et al., 2006; Habib y Jungthirapanich, 2010).

Además, la aplicación de la cadena de valor (vinculación con sociedad) en la educación superior puede mejorar la eficacia en los resultados de ES, profesionales y proyectos/investigaciones, con el mayor valor posible para la sociedad. Asimismo, es necesario el uso eficiente de sus dos cadenas de suministro (docencia y proyectos/investigación), para que produzcan profesionales y salidas de proyectos/investigación de calidad, respectivamente.

Por otro lado, en relación a otros sectores, el de servicios de educación presenta algo peculiar, dado que su producción es bidireccional (fluye en ambas direcciones), por la dualidad demandante-proveedor de insumos. Por ejemplo, el proveedor principal de su insumo más importante es asimismo su demandante mayoritario: cuando un estudiante demanda ser un profesional de ES (se beneficia/usa el servicio de ES), al mismo tiempo se debe poner en la línea como el principal insumo que se puede transformar en dicho profesional al final del proceso de ES (Sampson, 2000, Habib y Jungthirapanich, 2010).

Lo extraño es que muchas universidades (todas a nivel internacional ${ }^{2}$ ), se llegan a enfocar principalmente en enseñar e investigar modelos de cadenas de valor y/o de suministro que puedan mejorar la manufactura y los negocios en general. Sin embargo, muy pocas de dichas universidades (ninguna a nivel nacional) hacen sus propias actividades académicas alrededor de las cadenas de valor y de suministro de ES, a pesar de que dichas cadenas representan conceptos internacionales, con dimensiones uniformes a nivel global, que pueden ser aplicados en la ES (Habib y Jungthirapanich, 2008a, 2008b). 
Más allá de la perspectiva innovadora de las cadenas de valor y de suministro aplicadas en la educación superior, se plantea una cadena tanto orientada por la demanda de profesionales y proyectos de ES, así como integrada totalmen$t^{3}$. Por ello, se deben estudiar no sólo elementos de la demanda (cadena de valor), para lograr una integración hacia adelante (salida de los resultados terminados de ES), sino también elementos propios de la oferta (perspectiva tradicional de la logística de educación), que permite la integración hacia atrás (cadena de suministro), mejorando el proceso de entrada de insumos de ES. Todo ello para que la ES opere como un solo sistema de valor y de suministro, con una integración balanceada de las cadenas de valor y de suministro de la ES, con sus proveedores de insumos (lado de la oferta o entrada) y demandantes/beneficiarios (lado de la demanda o salida). En otras palabras, considerando las diferencias fundamentales entre la cadena de suministro (basada en la oferta) y la cadena de valor (basada en el beneficiario/demanda), se busca unificar tanto los elementos propios de la oferta tales como la integración proveedor de insumos-ofertante de ES, mejora de eficiencia y reducción de desperdicios, como los elementos propios de la demanda tal como crear un mejor valor a los ojos del demandante y la sociedad, que atraiga mayores flujos de fondos financieros y principalmente de nuevas peticiones de formar profesionales y realizar proyectos (reflejados en nuevas entradas tanto de estudiantes, como de proyectos e investigaciones, y sus colaboradores/patrocinadores).

Por parte de la demanda, se sabe a priori que los resultados y prestaciones de los profesionales e investigaciones de ES varían según la disciplina y grado académico, la institución de educación superior (IES) y otros factores endógenos y exógenos de la ES. Además, los empleadores pueden tener una gama de experiencias, procesos y prácticas para poder contratar los tipos de profesionales que requieren ${ }^{4}$. Lo mismo se puede decir de la articulación de la demanda de investigación, entre cooperantes/organizaciones y la investigación, desarrollo e innovación (I+D+i) que requieren. Ambas articulaciones de resultados de ES (docencia e investigación) son clave en permitir la sistematización de la cadenas de valor y de suministro de la ES integrada hacia adelante por la demanda. De la misma forma, por el lado de los insumos de la oferta, una IES tiene recursos de aprendizaje acumulados, que pueden permitir establecer indicadores y prácticas de articulación con la EM, que midan, entre otros, competencias requeridas de los graduados de EM por áreas de conocimiento. Por el lado de los insumos de la oferta de investigación, se debe establecer canales adecuados de comunicación y articulación entre IES's, cooperantes, patrocinadores, organizaciones de investigación (incluidos distintas organizaciones recipientes de bases de datos) y la sociedad, para estabablecer propuestas de problemas, formulaciones de desarrollo e innovación y recursos requeridos como materias primas de investigación. 
Nuevamente, ambas articulaciones de insumos de la oferta de ES (docencia e investigación) son fundamentales a considerar en la integración hacia atrás de las cadenas por la oferta.

Por otro lado, a pesar de las existentes preocupaciones de una articulación entrada-ES-salida, los retornos económicos personales por adquirir cualificaciones de ES y los retornos de desarrollo e innovación por la investigación y proyectos, aunque variables, son buenos en general y parece que continuarán con una creciente oferta. Pero ello no debe permitir que se desvíe la atención de realizar un estudio que pueda establecer modelos para un sistema de valor y de suministro, que incluya a la cadena de valor y de suministro de ES e integre intra e inter-institucionalmente el flujo mayor de actividades, desde su entrada de insumos de estudiantes de EM y proyectos por los suministradores/ proveedores (oferta) hasta su salida de profesionales y proyectos para los beneficiarios/demandantes (demanda).

Como punto de partida, así como los empleadores pueden variar su demanda de profesionales de educación superior (PES), las organizaciones pueden variar su demanda de necesidades de I+D+i. De la misma forma, se pueden tener formas variadas de vinculación con las IES, para permitir el intercambio de información sobre su demanda. Desde el lado de la docencia, la vinculación de las IES debe ser con los empleadores, para las clases de profesionales específicos (especializados o multidisciplinarios), y/o que posean habilidades y conocimientos más genéricos que les gustarían en todos los profesionales, y/o para cursos o talleres de la actividad del aprendizaje que las Instituciones de Educación Superior (IES) podrían suministrar para los empleadores. Desde la investigación, la vinculación debe ser con las organizaciones y cooperantes que puedan requerir el desarrollo de iniciativas de investigaciones y/o propuestas de proyectos conjuntos con las IES's. Además, las IES's pueden tener también un rango de puntos de contacto ${ }^{5}$ con empleadores y cooperantes, donde pueden obtener la información de la demanda. Dicha interfaz puede tener resultados numerosos tales como la planificación, la actualización curricular (planes de estudios) y de líneas de investigación, el desarrollo de nuevos cursos y proyectos, etc. La articulación de la ES con los empleadores y con los cooperantes, debe a su vez tener un impacto en la articulación de la ES con proveedores de insumos de estudiantes y de proyectos, respectivamente, debido a que las universidades deben ajustar su oferta conforme la realimentación de la demanda. Dicho ajuste implica variaciones en la demanda de insumos, tales como competencias de graduados de EM y materias prima para la investigación. Por tanto, es necesario otro interfaz de intercambio de información tanto de forma directa entre proveedores de insumos y universidades, como de forma indirecta, a través de la Secretaria de EM, colegios profesionales de EM, ONGs, etc. 
Actualmente, se pueden hacer suposiciones sobre cuán bien articulada y usada está la información de la demanda y oferta de profesionales e investigaciones de ES (PIES). Sin embargo, no existe evidencia empírica o teórica nacional de cómo trabajan dichos procesos y qué tan eficaces son en desarrollar y preparar los planes de estudios y de investigación, las cualificaciones y los nuevos programas de ES, así como asegurar los insumos necesarios para tales profesionales e investigaciones de ES. Aunque pueden existir casos aislados, no hay un tipo de evaluación sistemática, que pueda ser una base para el desarrollo futuro de políticas. Por ejemplo, algunos estudios internacionales muestran claros beneficios de empleadores y estudiantes cuyas universidades tienen mayores vínculos con empleadores. Sin embargo, dichos estudios no mencionan los diferentes tipos de articulaciones, o la eficacia en reformar el currículo de disciplinas y grados académicos por el mayor involucramiento universidadesempleadores, o los beneficios de proyectos/investigaciones conjuntas IESsociedad (Harvey, 2002; Mason et al., 2003; Brown y Hesketh, 2004). A pesar de que existen percepciones erróneas de las demandas de PIES por parte de las universidades $^{6}$ y confusiones por mensajes contradictorios de los empleadores (Dawson et al., 2006), existen casos donde las IES han sido exitosas en cumplir con las necesidades de los empleadores, tales como programas vocacionales de grados y posgrados conjuntos y educación profesional continua (educación a lo largo de la vida) ${ }^{7}$. Asimismo, existen propuestas internacionales para vincular EM-ES, por medio de la empleabilidad (Ward y Pierce, 2003), de políticas ${ }^{8}$ y de otros medios tales como evaluación y reformas de EM, entre otros (Brown y Niemi, 2007; Domina, 2007). Sin embargo, la literatura de las cadenas de suministro y de valor de investigación presenta poca evidencia sistematizada de articulación entrada-investigación de ES-salida (Habib, 2010).

A pesar de todo lo anterior, existe poca evidencia empírica del proceso o de las mejores prácticas de la cadena de valor y de suministro de la ES. Puede haber tensiones entre las IES, tratando de agregar valor a los insumos disponibles (ej. responder a la demanda educativa del resultado primario, el estudiante ${ }^{9}, \mathrm{y}$ del resultado secundario, la investigación) a los menores costos, y al mismo tiempo tratando de resolver las necesidades de los principales beneficiarios de la ES dentro de la sociedad (ej. responder con la oferta del profesional a la demanda del empleador y con la oferta de un investigación implementada en la sociedad). Ello puede verse reflejado en fallas o fricciones en la inteligencia del mercado, o en los vínculos con demandantes y/o con proveedores de insumos, o la percepción de los beneficiarios demandantes por los PIES y de los estudiantes (insumo de docencia) por los mejores (crecientes e interesantes) trabajos y profesiones. Además, pueden existir tensiones entre los puntos de vista expresados por los proveedores de insumos, en no entregarlos conforme las exigencias de las IES, y por las perspectivas de las necesidades de los beneficiarios demandantes (empleadores y sociedad), que pueden ser relativamente 
reducidas y a muy corto plazo, y lo que las IES ven como su papel y propósito legitimo en mantener estándares rigurosos de educación, equipando estudiantes con competencias y resultados de aprendizaje, y fortaleciendo a profesores y estudiantes con habilidades investigadoras y resultados de investigación, que tienen mayores beneficios a largo plazo para ellos.

Por otro lado, los colegios y gremios profesionales también pueden jugar un papel importante en contribuir a determinar la demanda de PIES para las IES's (en algunas disciplinas, sus colegios tienen un papel mucho mayor que la mayoría de los empleadores tales como el colegio de abogados, el colegio de médicos, etc.), así como en promover el desarrollo profesional entre sus miembros y las licencias o permisos para ejercer como profesionales. Existen variaciones entre qué tanto son una fuerza o un freno para la progresión. Asimismo, hay un papel que se le puede dar a la Secretaría del Trabajo y a las asociaciones sectoriales (industriales y de comercio) sobre el desarrollo y la innovación, donde algunos vínculos con las IES pueden desarrollarse más que otros. Por el lado de los proveedores de insumos de la oferta, más allá de las universidades como receptoras de éstos, se encuentran varios actores, entre otros, la Secretaria de EM, colegios de EM, gremios de maestros, Congreso Nacional, que deben jugar un papel importante en articular la entrada de insumos y los ofertantes de ES, de acuerdo a las necesidades de una ES orientada por la demanda de PIES, transmitidas por las mismas universidades. Algunos de dichos actores, tales como instituciones del gobierno, pueden contribuir en propuestas de iniciativas que mejoren y articulen todo el proceso proveedor de insumosES-demandante.

Así pues, el presente estudio establece fundamentos que permiten contribuir en la adecuada articulación entrada-ES-salida al centrarse en la siguiente pregunta de investigación: ¿Existe un modelo sistémico que integre las cadenas de valor y de suministro, permitiendo articular la ES con sus proveedores en la entrada de insumos de ES, así como con sus beneficiarios demandantes en la salida de los resultados de ES, y mostrando una mayor eficiencia y eficacia en las operaciones de las funciones fundamentales de ES: docencia, investigación y vinculación con la sociedad? Ello se contesta por medio de una investigación exploratoria, donde se busca estructurar e identificar relaciones básicas entre los conceptos mencionados, proponiendo un modelo que mida la integración de la ES con todos los actores involucrados. Dicho modelo prepara el camino para el estudio empírico que permita futuras investigaciones descriptivas y causales.

Así pues, la siguiente sección (2) presenta una revisión de la literatura. En el epígrafe 3, se hace una propuesta de un modelo sistémico de la cadena de valor y de suministro. El apartado 4 explica la metodología que debe seguirse para el desarrollo de una investigación empírica que gire alrededor de dicho modelo propuesto. Finalmente, la sección 5 establece algunas conclusiones y 
consideraciones finales, resaltando las implicaciones y futuras investigaciones por desarrollar.

\section{REVISIÓN DE LA LITERATURA}

El enfoque principal de los estudios de las cadenas de valor y de suministro ha sido en los negocios, sobre aspectos tales como reducción de costos y desperdicios, valor percibido y añadido, reducción del tiempo de respuesta, etc., entre los distintos grupos involucrados. La Figura 1 muestra la evolución que los estudios de la cadena de valor (Porter, 1985) y de la cadena de suministro han tenido desde sus inicios, para la industria manufacturera (ej. Ballou et al., 1984; Hammer, 2006) y, con mucho menos investigación, para el área de servicios (Fernie, 1995). Sin embargo, en años recientes, investigadores de la cadena de valor (Stylianou y Turner, 1999) y de suministro (O'Brien y Kenneth, 1996) ${ }^{10}$ han empezado a extender unos pocos estudios del área de servicios a IES's ${ }^{11}$ (Habib, 2010a).

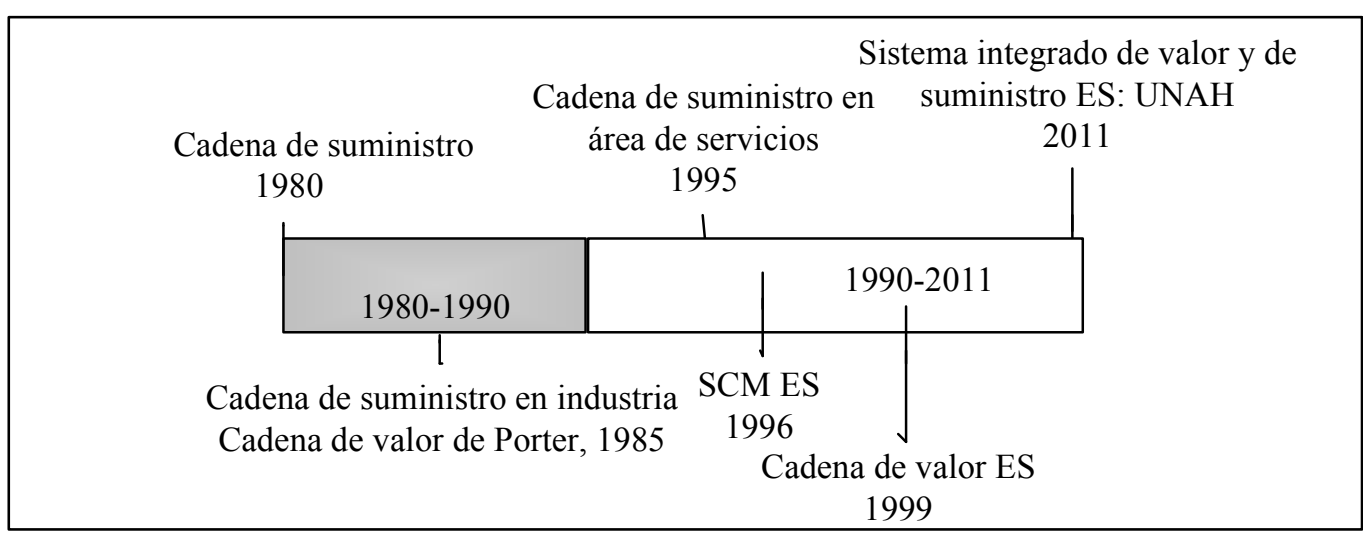

Figura 1. Antecedentes de un Sistema Integrado de Valor y de Suministro de ES

Sin embargo, la investigación de un sistema integrado de valor y de suministro de ES (que articule la ES tanto a los proveedores de insumos de ES como a los beneficiarios de los resultados de ES), es aún más escasa. Hay unos cuantos estudios sobre la cadena de valor, basados en la propuesta de Porter (1985), pero que no presentan una integración total, que incluya a los proveedores de insumos de ES y a los beneficiarios de los resultados de ES (Stylianou y Turner, 1999; Sison y Pablo, 2000; Tyran y Coakley, 2000; Elloumi, 2004; Van der Merwe y Cronje, 2004; Gabriel, 2006; Roldán y Hervás, 2007; Hansen, 2008; Mora et al., 2008; Pathak y Pathak, 2010). Finalmente, hay unos pocos autores que lo investigan desde una perspectiva exclusiva de la oferta, es decir la cadena de suministro.

Los insuficientes estudios de la cadena de suministro de ES no han ido más allá de entrevistas con estudiantes y empleados, con una referencia a la idea de 
la cadena de suministro ES (O’Brien y Kenneth, 1996). Por su lado, Lau (2007), presentó un estudio de caso con dos cadenas de oferta (estudiantes e investigación). Por otro lado, Habib y Jungthirapanich (2008a, 2008b, 2009a, 2009b, 2009c, 2009d, 2010, 2010a, 2010b) desarrollaron un modelo de la cadena de suministro integrando las cadenas de Lau.

Así pues, tomando las dos cadenas (valor y suministro) propuestas en este documento, a continuación se señalan algunos antecedentes sobre la cadena de valor y luego sobre la cadena de suministro. Ambas cadenas se desarrollan hacia la integración de un único sistema de valor y de suministro.

\subsection{Flujo de demanda en un sistema de valor y de suministro}

El estudio de la cadena de valor permite ver uniformemente a la ES, sus proveedores y demandantes (Porter 1985). Además, tal perspectiva de valor le da a la ES un mejor entendimiento de cómo sus diferentes unidades funcionan juntas para ofertar eficazmente de acuerdo al flujo de demanda al:

- Reconocer que las actividades dentro de una organización no son independientes sino interdependientes.

- Ayudar a encontrar áreas de mejoras para añadir los mayores valores posibles, dando la mejor percepción de los resultados a los demandantes.

E\&A IIES
Asimismo, la cadena de valor ayuda también en cómo lograr metas:

- Los servicios provistos pueden no ser la venta de productos, pero siguen siendo importantes.

- La cadena de valor puede ayudar a obtener lo máximo de los recursos que puedan ser limitados.

Además de que puede mostrar cómo la ES puede mejorar cada enlace, la cadena de valor es cuasi universal para toda organización, proveyendo una forma de examinar los procesos internos e identificando aquellos procesos que pueden ser mejor provistos (que añadan más valor) por actores distintos a los actuales. Tomando Porter (1985), se pueden considerar los siguientes dos grupos generales de actividades, que pueden ser aplicados en un sistema de valor y de suministro (Figura 2):

1. Funciones fundamentales: involucradas directamente en la producción y entrega de productos y servicios. En la ES, existen tres funciones fundamentales: docencia, investigación y vinculación a sociedad, que deben suministrar profesionales e investigaciones de ES.

2. Actividades de apoyo: tienen el potencial para incrementar la eficacia y la eficiencia de las funciones fundamentales, pero no están directamente relacionadas a suministrar los servicios y productos (profesionales e 
e investigaciones de ES).

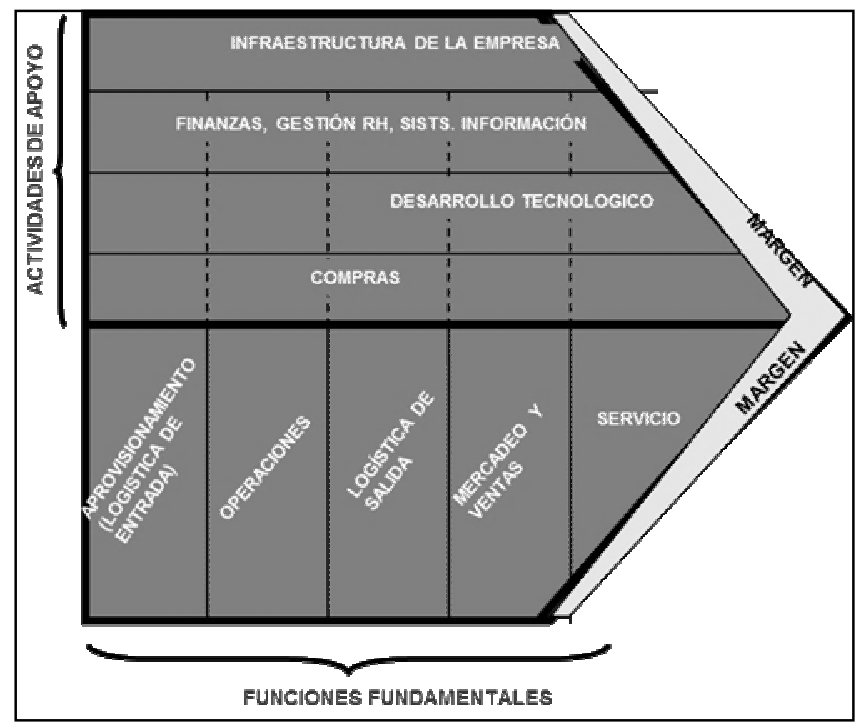

Figura 2. Funciones Fundamentales y Actividades de Apoyo del Sistema de Valor y de Suministro de ES

(Adaptado de Porter, 1985)

La Figura 2 distingue los siguientes componentes de las funciones fundamentales y sus equivalencias en ES:

- Logística de entrada: actividad que se encarga de recibir y almacenar los insumos. La distribución al área de producción (bienes y servicios) es según la necesidad.

- Captación, admisión, matrículas, propósitos de investigación, subvenciones.

- Operaciones: proceso que consiste en transformar los insumos en bienes y servicios terminados

- Docencia, investigación, consejería, tutoría.

- Logística de salida: almacenamiento y distribución de bienes y servicios terminados

- Graduación, publicación, práctica profesional, rendimiento.

- Mercadeo y ventas: identificación de necesidades de los demandantes y generación de entregas

- Captación y contratación, transferencia de tecnología y de conocimiento. 
- Servicio: apoyo post-entrega a usuarios de bienes y servicios como valor de servicio

- Apoyo académico, servicio a sociedad, apoyo a ex alumnos, apoyo D+i.

Asimismo, los siguientes componentes de actividades de apoyo (así como la evaluación de sus respectivos bienes) son parte de la Figura 2 arriba:

- Infraestructura e instalaciones: estructura organizacional, sistemas de control, gestión de administración, gestión financiera, etc.

- Gestión de recursos humanos: búsqueda de empleados, contratación, capacitación, desarrollo, y compensación (provisión para las unidades académicas y administrativas).

- Desarrollo de tecnologías que permitan apoyar actividades que agregan valor (gestión de TIC y otras tecnología, gestión de clases, gestión de recursos de investigación).

- Compras: adquisición y aprovisionamiento de insumos tales como materiales educativos (papelería, materiales de instrucción, etc.), suministro y equipo (mobiliario, computadoras, equipo de redes, etc.).

E\&A IIES

\subsection{Cadena de suministro de un sistema de valor y de suministro}

Un sistema de valor y de suministro de educación superior requiere coordinación e información entre todos los involucrados, que comparten procesos hacia adelante (beneficiarios como la demanda) y hacia atrás (proveedores de insumos de ES como parte inicial de la oferta). El uso de las TIC permite la posibilidad de diseñar un sistema de valor y de suministro coordinado para cumplir los objetivos de las IES, estableciendo relaciones eficaces y eficientes, tanto dentro como fuera de las IES (Sandelands, 1994).

Para ello, se puede establecer que el objetivo primordial de tal sistema debe ser mejorar la condición de resultados que la ES presta a sus demandantes (ej. profesionales, sociedad, etc.) (Habib y Jungthirapanich, 2008a). Por lo que las IES necesitan tener un grado de conocimiento acerca de los involucrados en el sistema (proveedores, IES y beneficiarios/demandantes), de cuya coordinación depende el rendimiento del servicio de la ES (logro de resultados deseados de las cadenas).

Así pues, siendo el conocimiento (producto fundamental de la educación) intangible, se diferencia de cualquier producto de la industria manufacturera. 
Además del conocimiento, otra diferencia general entre ambas es que la educación eficaz y eficiente se basa mucho en la experiencia y la ética personal. Otro punto es la definición misma para dicho sistema, como se dijo en la sección anterior, contrario a lo que fácilmente definible sería un sistema manufacturero de valor y de suministro (donde cada participante en la cadena recibe insumos de un conjunto de proveedores, entonces procesa dichos insumos para entregar los resultados a un conjunto de demandantes), un sistema de ES es un poco más compleja. Además de presentar los mismos tipos de actores, el sistema de ES define también un flujo bidireccional por una dualidad proveedor de insumos-demandante, dado que algunos de sus proveedores principales de insumos (oferta) son simultáneamente demandantes (demanda), y viceversa, algunos demandantes tienen que proveerse a sí mismos como insumos a los procesos de servicios de ES (Sampson, 2000). Específicamente, los estudiantes demandan ser profesionales (usuario potencial), y/o algunos resultados terminados de ES (profesionales y proyectos ${ }^{12}$ ) se pueden proveer ellos mismos para más procesos de ES. Además, otro caso especial es que la EM requiere profesionales y proyectos de ES para graduar sus estudiantes que serán los insumos de ES, lo que deja a la ES como un ofertante de resultados que sirven para preparar sus propios insumos. Otros casos de proveedor-demandante son patrocinadores de becas, de investigaciones, las mismas IES's, etc. Para más detalles de los actores del sistema ver Figura 3 y la siguiente sección.

Figura 3. Oferta y Demanda: Actores en un Sistema de Valor y de Suministro

\section{Valor (eficacia): fondos, información, pedidos}

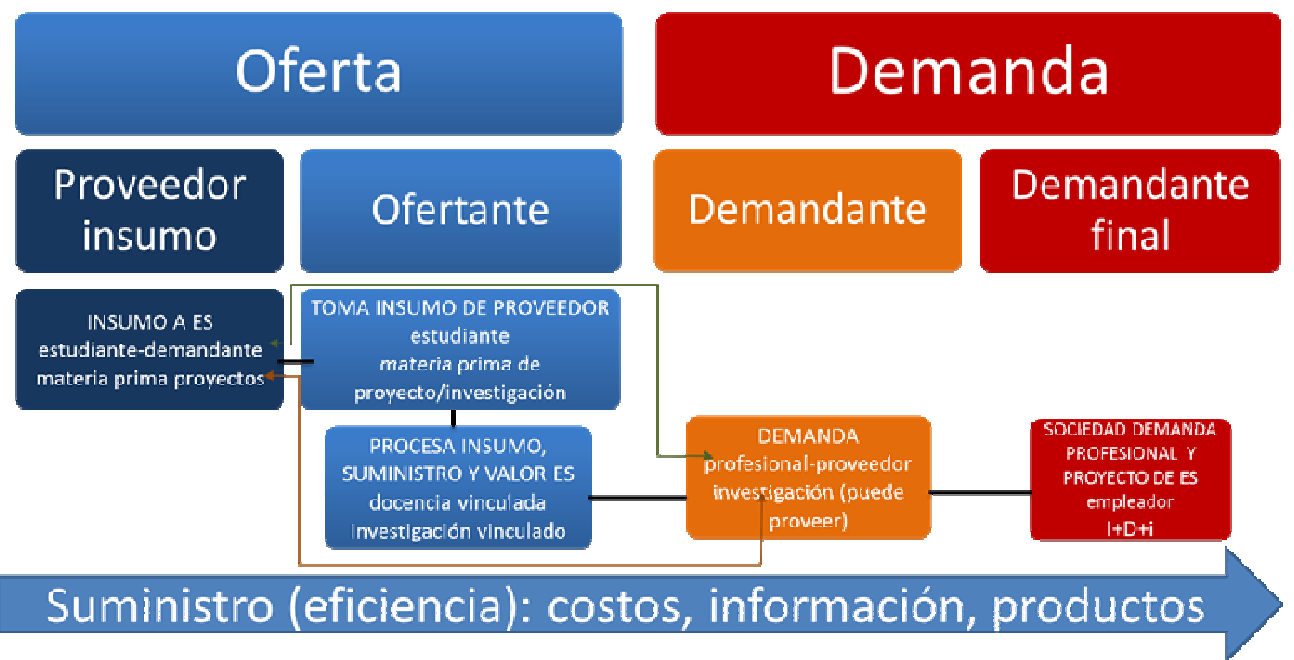

12 En el presente estudio, los términos proyecto e investigación se intercambian entre sí, expresando la segunda función fundamental de ES de investigación, que en este contexto abarca distintos tipos de proyectos e investigaciones de ES. 
Por otro lado, en los estudios de la cadena de suministro, se puede ver que tanto la industria manufacturera como la de servicios tienen estrategias similares para operar la cadena de suministro (Fernie y Clive, 1995; Sengupta y Turnbull, 1996; Nixon, 2001). Ello se establece con una serie de tres niveles de decisiones, caracterizada en modelos de negocios: estrategias, planificación y operaciones (Figura 4). Dichos niveles de decisiones se pueden adoptar en el presente estudio sobre un sistema de valor y de suministro de ES (Harris, 1998):

- Estrategias: son las decisiones al más alto nivel, como parte del área de planificación de acciones. Tienen un efecto a largo plazo en la cadena de valor y de suministro. No se requiere una respuesta rápida, ya que a este nivel las decisiones no se toman día a día. La Alta Dirección es tanto el tomador de decisión como el usuario de esta información.

- Planificación: tiene las decisiones a nivel medio, que apoyan las decisiones estratégicas. Tienden a ser a mediano plazo y de significancia media, con consecuencias moderadas.

- Operaciones: son decisiones de nivel bajo, que se encuentran en el área de planificación y programación operacional. Están limitadas por las decisiones estratégicas y las de planificación. Son de corto plazo, porque a este nivel es indispensable una respuesta rápida, que afectan al día a día.

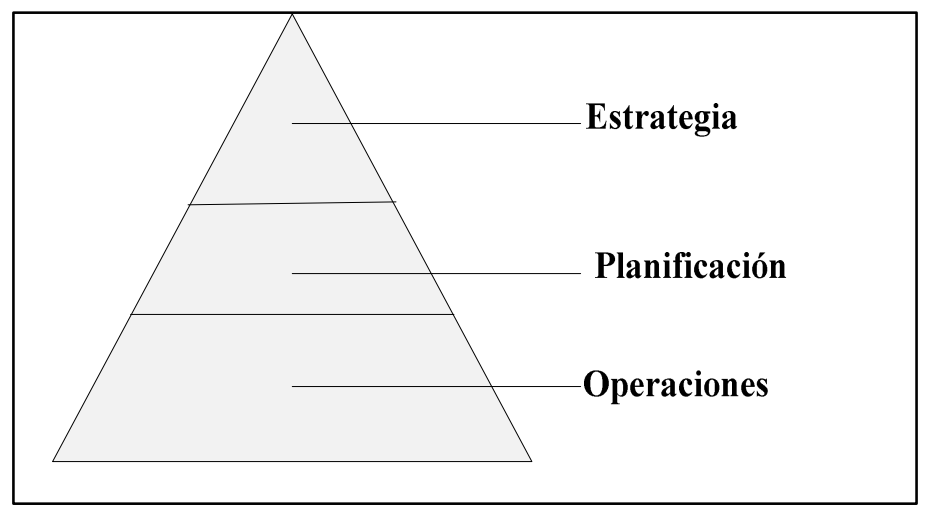

Figura 4. Decisiones en un Sistema de Valor y de Suministro (Adaptado de Habib y Jungthirapanich, 2008b) 


\section{MODELO CONCEPTUAL DE INVESTIGACIÓN}

Basándose en la producción, gestión y transferencia del conocimiento, pueden existir dos servicios principales de una cadena de suministro de ES (CSES): docencia e investigación. La primera establece servicios directos e indirectos de docencia para procesar insumos (ej. graduados de EM, estudiantes de postgrado, necesidades de talleres, de capacitaciones, de diplomados, etc.). Los servicios directos pueden incluir diseño y desarrollo, fuente y selección, formación académica y no-académica, formación práctica y vocacional, formación de grados y posgrados, evaluación de resultados, desarrollo de educación continua (a lo largo de la vida), etc. Los servicios indirectos pueden ser la mejora y mantenimiento del campus, la infraestructura de las TIC, la oferta de residencias universitarias, las autorizaciones de acceso a instalaciones e información (dura y digital), bibliotecas, librerías, seguridad, restaurantes/comedores, instalaciones deportivas, etc. (Lau, 2007). Para asegurar la calidad, una CSES requeriría procesos personalizados para cada estudiante. Sin embargo, debido a que cada estudiante es diferente y ninguna IES podría establecer un proceso de CSES individual para cada estudiante, en el diseño y desarrollo crítico de cada estudiante, se debe asignar un asesor académico que supervise el proceso de desarrollo del estudiante por medio de la CSES (Habib y Jungthirapanich, 2010).

La segunda, investigación, casi siempre cara y a largo plazo, requiere de una CSES personalizada, proactiva y reactiva para satisfacer a los demandantes. En primer lugar, la CSES debe gestionar la comunicación a profesionales e instalaciones en una universidad para prevenir duplicaciones cuando se enfoque en investigaciones básicas, que necesiten desarrollar observaciones con encuestas que recojan datos relevantes. En segundo lugar, cuando se necesite desarrollar algún sistema y/o tecnología para un sector en particular, la CSES debe buscar no sólo a los actores relevantes (que sean profesionales en investigaciones aplicadas), sino también a las instalaciones necesarias, para que ambos, profesionales sea posible realizar una investigación de forma más eficaz y eficiente (Habib y Jungthirapanich, 2010).

Con lo que podría ser considerado un tercer servicio primordial junto y paralelo (pero en dirección contraria) a las dos CSESs anteriores (docencia e investigación), una ES integrada necesita de otros procesos personalizados, proactivos y reactivos, que permitan realimentar el nivel de satisfacción de los demandantes y la sociedad por el valor percibido de los resultados de los servicios actuales de la ES, en todos los niveles geográficos posibles: local, regional, nacional e internacional. La idea del tercer servicio es una vinculación con sociedad dentro de una cadena de valor de ES (CVES) que permita monitorear el valor

E\&A IIES 
añadido por el proceso de ES, desde la condición inicial de los insumos hasta la recepción de los resultados de la ES por parte del demandante y la sociedad, evaluando la cantidad de valor añadido en cada paso de la ES. Para ello, la CVES debe construir una vinculación ES-sociedad que tome dicha realimentación para mejorar sus servicios de las dos CSES (docencia e investigación) y el nivel de vinculación, corrigiendo lo presente o desarrollando otras alternativas, de acuerdo a la necesidad. Además, dicha articulación por la CVES debe promover los servicios actuales como parte de ambas CSES y el desarrollo de nuevos servicios dentro de las mismas dos CSES, por medio del flujo de valor de la sociedad a los otros involucrados en ES (otros beneficiarios demandantes de resultados de ES, IES, proveedores de insumos de ES).

Sin embargo, para tener una mayor perspectiva completa, dicho flujo debe ir más allá de uno direccional de valor por la CVES (demanda-oferta), al integrar el flujo de suministro desde la otra dirección por la CSES (ofertademanda), que resulte en un sistema integrado de valor y de suministro de ES (SIVSES), cuyo flujo sea por tanto bidireccional. En dicho flujo del proceso del SIVSES, las universidades deben trabajar en diseñar los currículos en colaboración cercana con diferentes entidades, tales como colegios, institutos tecnológicos, estudiantes actuales de ES, personal universitario, empleadores, profesionales, cooperantes, usuarios de sus proyectos/investigaciones, etc., para asegurar que satisfagan las necesidades de todos los participantes en el SIVSES (Heskett, 1964). Además, el SIVSES debe tener una visión dirigida por los demandantes de resultados de ES, que puede producir un número de ventajas competitivas a la ES al ayudar a mejorar la productividad y la satisfacción del demandante, y a producir resultados de calidad. La gestión de la calidad de ES muestra beneficios potenciales de asociar los resultados finales con los proveedores de insumos (Habib y Jungthirapanich, 2010a).

Por todo ello, se requiere un punto de vista holístico del marco conceptual del SIVSES, formulado de forma multinivel y bidireccional. Siendo sistémico, el sistema necesita de todos los involucrados para lograr los resultados finales eficientes y eficaces. Para ello, se establecen siete componentes significativos del modelo propuesta para el SIVSES: los insumos, el proceso de la ES (funciones fundamentales y actividades de apoyo), tres tipos de resultados (primarios, secundarios, terciarios) y el vínculo sistematizado de la sociedad a los insumos y al proceso. Dicho modelo integrado del sistema puede cumplir mejor los requisitos de los dos tipos de demandantes: 1) demandantes intermedios (profesionales/instruidos y resultados de investigación); y 2) demandante final (sociedad, empleadores, sectores de proyectos de investigación, desarrollo e innovación (I+D+i) ejecutados/implementados).

El proceso de ES (usando sus dos tipos de funciones fundamentales como suministro) en la Figura 5 recoge los insumos de ES (graduados de EM como estudiantes y materias prima de proyectos) y busca transformarlos de la 
manera más eficiente en resultados primarios finales tales como profesionales y resultados de investigación/proyectos. Algunos profesionales pueden decidir trabajar como docentes o administrativos de la universidad o en otra universidad (resultados terciarios al proceso de funciones fundamentales y de actividades de apoyo $)^{13}$. Mientras que, como resultados secundarios, otros profesionales pueden buscar el estudio de posgrados y algunos resultados de investigación pueden ser considerados como insumos en nuevas rondas de investigación de ES, en la misma u otra universidad ${ }^{14}$. Estos resultados secundarios, al mismo tiempo como proveedores de insumos, muestran claramente una dualidad salida-entrada. Ello puede representar la oportunidad de insumo como parte del proceso de ES, al tener la posibilidad de proveer conocimiento, habilidades, prácticas y experiencia de estudiantes de grado para instruir estudiantes de pregrado y para gestionar proyectos (insumo de postgrado para procesar insumo de pregrado). En todo ello se busca también la articulación de la sociedad con los resultados, procesos e insumos de la ES, que signifique la búsqueda de una mejor percepción del valor de los resultados de ES, por el mayor valor añadido en cada parte del sistema.

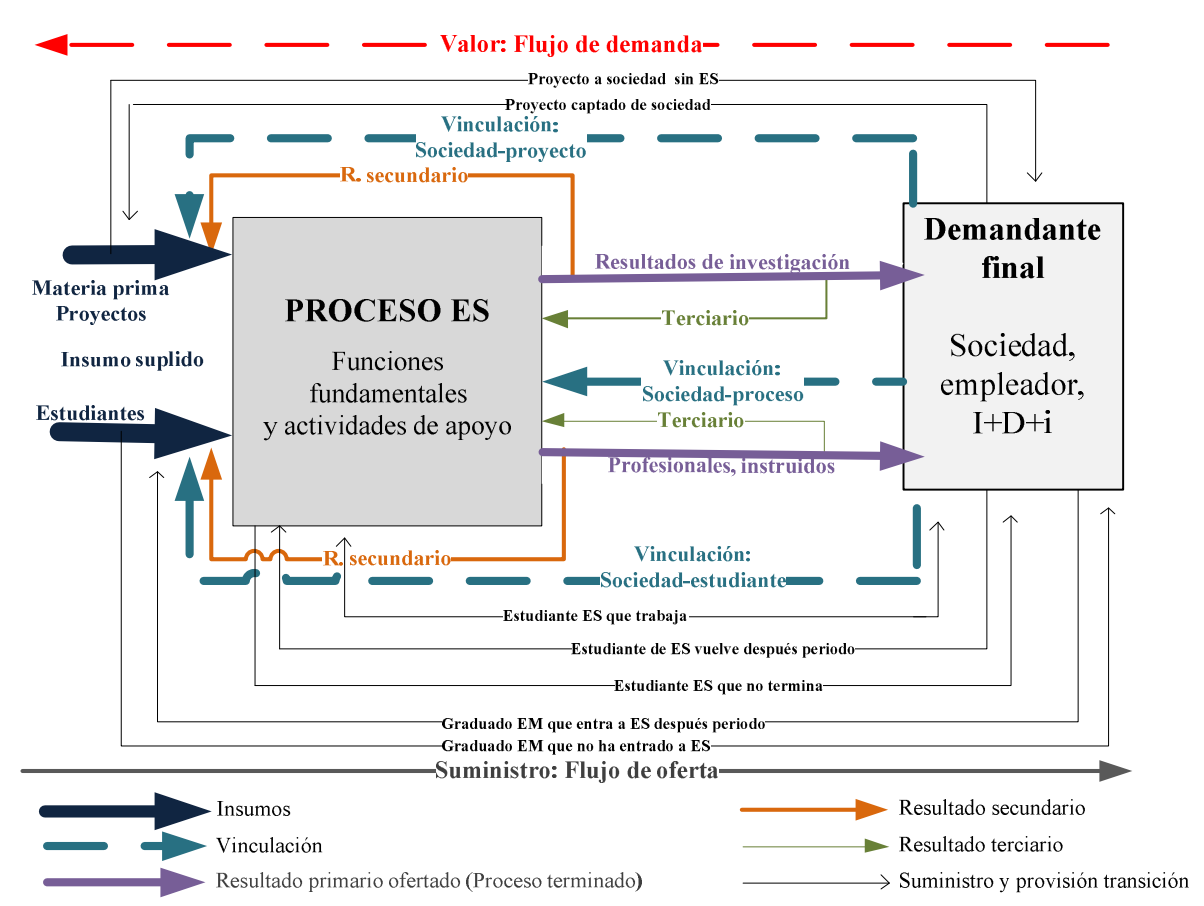

Figura 5. Modelo Propuesto: Sistema Integrado de Valor y de Suministro de ES (Adaptado de Habib, 2010a) 
Un buen factor de medición en la aplicación de los tres niveles de decisión descritos (estrategias, planificación y operaciones) ${ }^{15}$, que interrelacione los elementos del sistema pudiera ser la clave para que los tres tipos de resultados (primarios, secundarios, terciarios) reúnan las expectativas de los involucrados. Finalmente, lo más importante es recordar que la fuente de valor fluye del demandante, por lo que es necesario considerar el flujo de demanda, que va de la sociedad hasta el proveedor de insumos, pasando por los resultados y el proceso/oferta de ES, como se ve en las líneas punteadas rojas (las líneas verdes de articulación con sociedad siguen también el flujo de valor). La figura enseña también lo que podría ser considerado como posibles fallas e indebida articulación en el sistema de ES, representados por líneas como provisiones y suministro en transición (ej. insumos potenciales que no entran en el proceso de ES, pudiendo o no entrar eventualmente; estudiantes como suministro de ES que tienen que trabajar dentro o fuera de ES y/o se tienen que retirar, pudiendo o no re-entrar de nuevo; etc.).

\subsection{Demanda de ES: usuarios de resultados}

Tomando como referencia el flujo de demanda de la Figura 3, se identifican tres grupos fundamentales del SIVSES: 1) los demandantes de los resultados

E\&A IIES de ES; 2) el proceso de ES con su cadena interna de suministro y de valor (CAS); y 3) los proveedores de insumos de ES.

\subsubsection{Demandante final: demandante de demandante (usuario de profesionales y proyectos)}

El presente estudio toma a la sociedad como el beneficiario/demandante de instancia final del SIVSES. Dado que la ES es parte intrínseca de la sociedad, los resultados finales de tal sistema (profesionales de calidad y resultados de investigación/proyectos de desarrollo e innovación de calidad) son entregados inevitablemente a la sociedad (principalmente a los empleadores y a sectores donde se implementan proyectos). Así pues, se pueden ver dos tipos principales de contribuciones a la sociedad (Habib, 2010): 1) el capital intelectual por profesionales e instruidos trabajando con todo tipo de empleadores (incluido emprendedurismo/autoempleo); y 2) proyectos de I+D+i de ES ejecutados/ implementados en sectores de la sociedad. 


\subsubsection{Demandantes intermedios: primeros usuarios}

El demandante intermedio es el que demanda el resultado de ES en primera instancia, antes de entregar dicho resultado a la sociedad. Se identifican dos tipos de demandantes intermedios: 1) estudiantes demandando docencia para ser profesionales de ES, y 2) universidad-colaboradores demandando investigación/proyectos. Ambos tipos de demandantes presentan una dualidad demandante-proveedor (Figura 6).

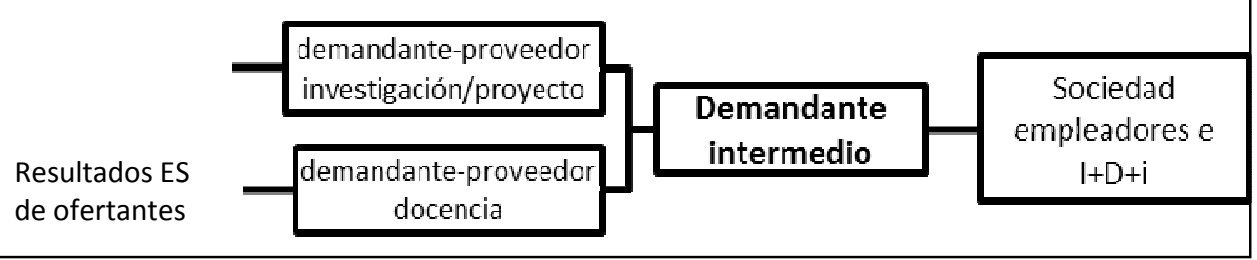

Figure 6. Demandantes intermedios de la ES

(Adaptado de Lau, 2007)

\section{Demandantes de docencia}

- Dualidad demandante-proveedor de insumos ${ }^{16}$ : flujo bidireccional que contribuye al desarrollo de profesionales.

- Donde graduados de EM demandan ser profesionales (todos los niveles de resultados)

- Cuando profesionales (graduados de ES) vuelven a demandar como estudiantes de postgrado o de otro tipo de instrucción (resultados secundarios)

- Cuando existe un patrocinador de becas, apoyo familiar, IES's etc. (todos los niveles de resultados)

\section{Demandantes de investigación/proyectos}

- Dualidad demandante-proveedor de insumos: flujo bidireccional por la obtención de resultados y conocimientos de $\mathrm{I}+\mathrm{D}+\mathrm{i}$ (investigadores, publicaciones, conclusiones, proyectos terminados, etc.)

- Para propia universidad, para organizaciones patrocinadoras y colaboradores de proyectos de $\mathrm{I}+\mathrm{D}+\mathrm{i}$, etc. (todos los niveles de resultados).

- Son necesarios de nuevo en rondas subsecuentes de proyectos. Aquí se identifican líneas y programas permanentes y longitudinales de investigación (resultados secundarios). 
Siendo los demandantes la fuente del flujo valor, sus percepciones son la principal referencia en el modelo propuesto, para lograr la mejor valoración. Para lograr resultados de ES más efectivos es necesario añadir los valores más altos en cada paso del sistema, y/o usar un enfoque de mercadeo y/o innovación en mejorar las percepciones de los demandantes.

Como caso especial, los demandantes que simultáneamente proveen insumos pueden servir para monitorear permanentemente valores añadidos por distintas etapas del proceso de ES, dado que pueden saber en qué condición dichos insumos estaban inicialmente. Por otro lado, cuando estudiantes de ES se gradúan, al hacer uso de autoevaluaciones, se pueden aproximar márgenes entre valores percibidos de ES y gastos para lograrlos.

\subsection{Ofertante de ES: proceso de suministro y de valor}

Lógicamente, los únicos ofertantes de ES son las IES's. Por lo que, como se definió en la sección 2 y la Figura 5 más arriba en esta sección, el proceso de una IES tiene varias actividades de apoyo y tres funciones fundamentales en tres cadenas: dos cadenas de suministro (funciones de docencia y de investigación) y una cadena de valor (función de vinculación a sociedad). La Figura 7 muestra los componentes del proceso de ES desde una perspectiva de suministro y de valor.

E\&A IIES

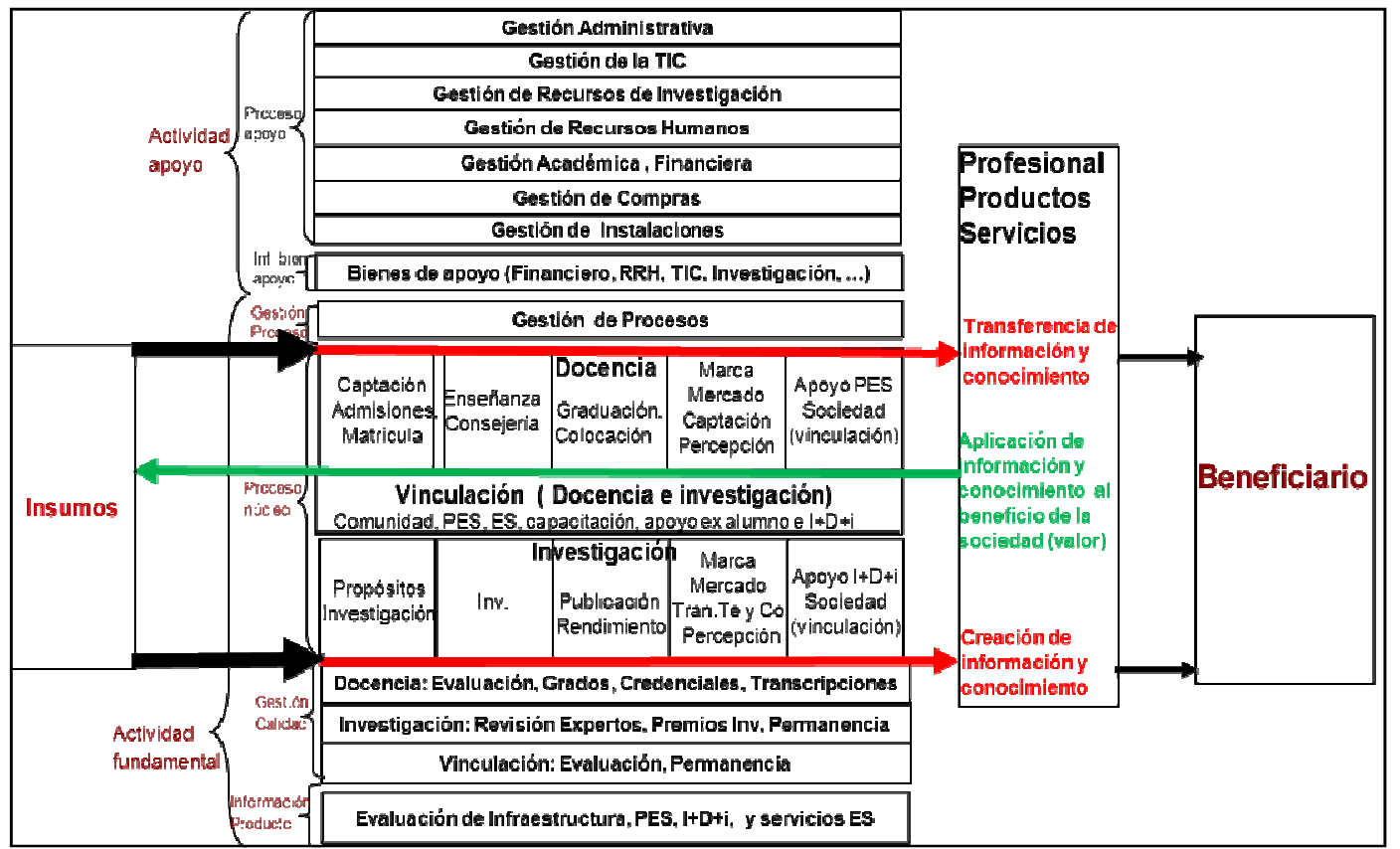

Figura 7. Componentes de la Cadena Interna de Suministro y Valor (Adaptado de Porter, 1985) 
Además, hay otros aspectos importantes, específicos de la ES, a considerar como parte de las funciones fundamentales, tales como:

- Gestión de procesos.

- Procesos de las 3 funciones fundamentales de la ES

- Docencia: establecida en niveles de pregrados y postgrados, talleres, diplomados, certificaciones, instrucción, inducción, capacitación, empleabilidad y tasas de retorno, etc.)

- Investigación: por medio proyectos, I+D+i, aplicabilidad y tasas de retorno, etc.).

- Vinculación con la sociedad: función que permita eje articulador hacia la docencia y la investigación, considerando la percepción del valor de resultados de ES que tienen los profesionales, la sociedad, los empleadores y otros demandantes de ES.

- Gestión de calidad total en las funciones fundamentales

- Diferentes programas de evaluación de la calidad de la docencia e investigación.

- Evaluación del rendimiento de investigadores y docentes.

- Información de servicios

E\&A

IIES

- Capacidades de facultad: ranking administrativo y académico de la facultad, universidad, etc.

- Currículo de docencia e investigación y establecimiento de programas: facultades, escuelas, departamentos y carreras establecidos para desarrollo de docencia e investigación.

- Cultura universitaria tales como gestión por resultados, buena administración, excelencia en docencia e investigación, programas de investigación conjuntos, etc.

- Infraestructura/instalaciones académicas: que contribuyan a mejorar la docencia y la investigación, la evaluación de calidad de las mismas, etc.

Siendo el proceso de ES la parte principal del modelo propuesto, es importante considerar aquí todos los pasos que realmente contribuyen a generar los servicios que los demandantes valoran, para eliminar aquellos que no construyen. Con ello, el modelo puede reducir el desperdicio de los recursos, y aumentar el margen entre el valor de los demandantes y el costo de entrega, mejorando así la eficiencia. 


\subsection{Proveedores de insumos de ES}

Se identifican dos proveedores principales de insumos a la ES, a saber: 1) proveedores de docencia, y 2) proveedores de investigación/proyectos. Estos dan los insumos a los ofertantes de ES, quienes los procesan y los entregan como resultados de ES, a los demandantes intermedios en primera instancia y a la sociedad en instancia final (Figura 8).

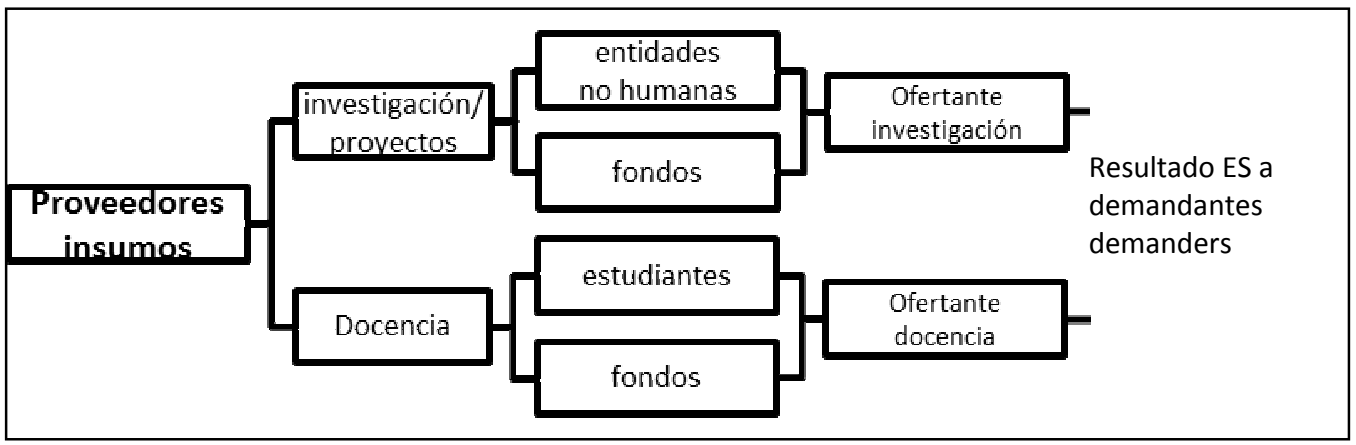

Figura 8. Proveedores de insumos de ES: docencia e investigación (Adaptado de Lau, 2007)

E\&A IIES

Proveedores de docencia:

- Proveedores de estudiantes: colegios, universidades, empleadores, organizaciones públicas y privadas, etc.

- Proveedores de fondos: autofinanciado, familia (padres, parientes), becas por universidades, gobierno y organizaciones privadas, etc.

Proveedores de investigación/proyectos:

- Proveedores de entidades no humanas

- Propuestas proyectos internos.

- Propuestas de proyectos externos.

- Propuestas de proyectos mixtos.

- Insumos especiales para investigación: acceso remoto a bases de datos de revistas científicas, diferentes clases de bases de datos, publicaciones, etc.).

- Proveedores de fondos

- Financiamiento interno de I+D+i por universidad. 
- Financiamiento externo de I+D+i por organizaciones públicas y/o privadas.

- Financiamiento mixto de I+D+i por universidad y organización externa

\section{CONCLUSIONES Y CONSIDERACIONES FINALES}

El objetivo principal planteado en el presente trabajo fue establecer un modelo sistémico integrado de las cadenas de valor y de suministro guiado por demanda, como fundamento para examinar detalladamente el fenómeno de integración total de ES (hacia delante por la demanda y hacia atrás por la oferta) en Honduras, que debe caracterizar a todas las partes de dicho sistema. Así pues, el modelo propone ambas cadenas de suministro principales de ES como las dos primeras funciones fundamentales de ES (docencia e investigación), que juntas deben articular no sólo el proceso entero de ES (las tres funciones fundamentales y actividades de apoyo), sino también los proveedores de insumos de ES por el lado de la oferta y demandantes de resultados de ES por el lado de la demanda, para tener un proceso de ES eficiente (más bajos costos y reducción de desperdicios en la oferta). Por ejemplo, las actividades que no contribuyen a cumplir las necesidades de los demandantes son desperdicios y no añaden valor. Por lo mejoras de procesos de de tareas y actividades que ocurren en el sistema de ES propuesto pueden mostrar y reducir o aun eliminar cualquier desperdicio en existencia. Al reestructurar procesos que generan los servicios de ES que los demandantes valoran, pueden gastarse menos recursos. Al mismo tiempo, el modelo se diferencia y va más allá de la literatura actual al presentar simultáneamente una cadena de valor por la vinculación a sociedad (la tercera función fundamental de ES), que si toma la ventaja de la articulación anterior de la oferta, la ES puede entregar los resultados más valorados, por medio de la realimentación de percepciones de valor desde los demandantes de ES, pasando por el proceso de ES, hasta los proveedores de insumos de ES. Dichas percepciones pueden obtenerse al añadir los mayores valores en cada paso no sólo del proceso de ES sino también de sus dos integraciones (adelante hacia sus demandantes y atrás hacia sus proveedores), y/o al usar enfoques de mercadeo e innovación, para mejorar la percepción del demandante, haciendo por tanto resultados de ES más eficaces. Por ello, este sistema que alinea las cadenas de valor y de suministro pueden entonces tener una influencia en el balance entre la oferta y demanda de resultados de ES: profesionales e investigación de ES.

Resumiendo lo anterior, para que la demanda de la sociedad pueda guiar a la

E\&A

IIES

43 
oferta de competencias de profesionales de ES, y de productos y servicios de investigación de ES, se deben buscar y operar mecanismos (y ser ampliamente usados) orientados por los mismos demandantes (ej. Capacitar para Ganar ${ }^{17}$ ), logrando lo siguiente: 1) articulación ofertante de ES-proveedor de insumos (ej. EM y cooperante); 2) articulación ofertante de ES- demandantes (ej. empleadores y sociedad); y 3 ) asociaciones profesionales, industriales y de comercio, y otros organismos de la sociedad civil con un papel más influyente en ayudar a desarrollar un sistema de valor y de suministro de ES más proactivo, reactivo (cuando sean necesario) y relevante.

Con ello, se podrán conocer más necesidades acerca de la demanda de PIES y cómo la oferta misma de PIES puede influenciar dichas necesidades:

- Información sobre la demanda de ES disponibles a las IES

- Demanda académica de carreras y grados de ES vs. demanda laboral de profesionales de ES (incluido proyecciones, empleabilidad y tasa de retorno).

- Demanda académica de investigación/proyectos vs. demanda del mercado de I+D+i de ES (incluido aplicabilidad y tasa de retorno).

- Verificación de si existe un intercambio sistemático ajustado para el propósito (maneras en que la demanda está disponible).

E\&A IIES

- Interpretación y uso de la información de la demanda por la ES (si demanda influencia oferta).

- Información sobre la oferta de ES, conforme la demanda de HE, disponible a los demandantes de ES

- Oferta de ES (incluido proyecciones)

- Insumos necesarios de proveedores para profesionales (estudiantes de EM) y para investigación (materia prima y recursos)

- Verificación de si existe un intercambio sistemático ajustado para el propósito (maneras en que la oferta está disponible).

- Interpretación y uso de la información de la oferta por demandantes (si comunican diferencias de necesidades de oferta).

- Información sobre la oferta de ES, conforme la demanda de ES, disponible a los proveedores de insumos

- Oferta de ES (incluido proyectada)

- Insumos necesarios de los proveedores de insumos para los profesionales de ES (estudiantes de EM) y para investigaciones de ES (materia prima $\mathrm{y}$ recursos). 
- Verificación de si existe un formato que se ajuste a un propósito.

- Interpretación y uso de la información de la oferta de ES por los proveedores de insumos.

- Obstáculo entre demandantes e IES, y entre IES y proveedores de insumos.

- En el dialogo mismo.

- Forma en que se usa la información.

- Demanda y oferta de ES más cercana a las necesidades descritas.

- Definición, redefinición (si se necesita) y adecuación entre oferta y demanda.

Así pues, un enfoque de estas características constituirá el desarrollo pionero en Honduras de la investigación relativa a un sistema integrado de valor y de suministro de ES, que busque la articulación de ES con los proveedores de insumos y con los demandantes de ES. Además, el enfoque colaborará a proveer el fundamento para un examen no sólo nacional, sino también mundial de la gestión del sistema integrado propuesto, así como para responder a un número de preguntas acerca de cómo balancear la oferta de ES con su demanda. Por tanto, dicho enfoque significará un importante examen de las cadenas de valor y de suministro de ES y sus resultados serán de gran interés para académicos, profesionales, empleadores y para la sociedad en general.

Aunque tal modelo se formuló desde un punto de vista teórico, se espera se pueda probar desde una perspectiva empírica en la segunda etapa lógica, ya en curso. Como punto de partida, el componente teórico del enfoque holístico permitió sintetizar la literatura del apoyo a la cadenas de valor y de suministro de ES, los proveedores de insumos y los demandantes de ES. Este fue un primer paso hacia la actividad de identificar y luego contactarlos para probar cualitativa y cuantitativamente por prácticas, relaciones, flujos de información, rendimientos, etc. entre todos ellos. Así pues, el componente empírico permitirá desarrollar una base de datos de información acerca de las operaciones de la ES en Honduras, en conjunto con todos los actores involucrados. Esta base de datos será entonces usada para probar proposiciones e hipótesis respecto al sistema integrado de valor y de suministro de ES y su relación con la oferta y demanda de investigación y de profesionales de ES.

La información de la base de datos por medio de encuestas puede servir para relacionar varias prácticas y procesos de gestión del sistema propuesto, verificando su integración total. Los datos pueden ser complementados con información cualitativa, recogida durante una serie de visitas a los involucrados, que incluirá entrevistas estructuradas y visitas por las instalaciones. 
Como paso previo, se ha seguido complementando el análisis de la literatura internacional, iniciada aquí, en relación con los aspectos teóricos que hacen operativo los tres niveles de decisión en el modelo del sistema integrado con las funciones fundamentales de docencia, investigación y vinculación, así como las actividades de apoyo. Con ello, se prepara el camino para la recogida de los datos y los posteriores análisis empíricos. Específicamente, a partir de dicho análisis bibliográfico y operativo, se podrán identificar ciertos factores de éxito, para su posterior desarrollo y comprobación estadística, que servirán de fundamento para un estudio empírico del sistema propuesto.

Por tanto, un estudio de campo es una aportación apreciable, ya que se espera encontrar el uso (consciente o no, parcial o total) de algunos de los fundamentos de la integración de las cadenas de valor y de suministro por parte de algunos de sus actores. Además, las lecciones aprendidas en dicha investigación se pueden propagar a todo el sector de la ES, a los empleadores, a los cooperantes, a los proveedores de insumos de ES y a la sociedad en general, por medio de la difusión de sus resultados.

Finalmente, aún si la demanda (ES-demandante) y la oferta (entrada-ES) estuvieran interpretadas y articuladas correctamente, existen algunos asuntos tales como los tiempos en desarrollar nuevos programas, o en hacer cambios curriculares, o en proponer premios de docencia y/o investigación, dado que la mayoría de las IES (y la EM particularmente) no son generalmente conocidas por

E\&A IIES su flexibilidad y velocidad de respuesta. Ello es comprensible dado la naturaleza habitualmente burocrática de los sistemas de educación (ES y EM), así como la necesidad de que las IES se aseguren de no sólo materializar los profesionales e investigaciones prometidos a los demandantes (ej. empleadores e implementación de proyectos en la sociedad), sino también recibir los insumos requeridos de sus proveedores (ej. graduados de EM y materia prima de proyectos de cooperantes).

Las prioridades de investigación futura propuestas en este documento requerirán de un adecuado financiamiento, una masa crítica a nivel nacional y otros recursos. En conjunto, ello puede facilitarse por un compromiso de la UNAH y las demás instituciones de ES, el Estado, los empleadores, los cooperantes y la sociedad en general.

\section{Reconocimientos}

Esta investigación ha sido financiada parcialmente por el Consejo Nacional de Educación de Honduras, la Vicerrectoría Académica de la UNAH, la Beca de Investigación de la UNAH 03-2011 y el proyecto DPI-2008-04788 del Ministerio de Ciencia e Innovación de España. Los autores desean agradecer el apoyo del Gobierno de Honduras, de la UNAH, y del Gobierno de España. 


\section{REFERENCIAS}

Ballou, R.H., Gilbert, S. y Mukerjee, A., 1984. New managerial challenges from supply chain opportunities. Industrial Marketing Management, 29 (1), $7-18$.

Brown, P. y Hesketh, A., The Mismanagement of Talent: Employability and the Competition for Jobs in the Knowledge Economy, Oxford University Press, Oxford.

Brown, R.S. y Niemi, D.N., 2007. Investigating the Alignment of High School and Community College Assessments in California. National Center for Public Policy and Higher Education.

CFE, 2009. Using demand to shape supply: An assessment of the higher level skills needs of employers in England. OECD.

Clemente, M.N. y Greenspan, S.D., 1998. Winning at mergers and acquisitions: The guide to market-focused planning and integration. John Wiley, New York.

Dawson, I., Jackson. A. y Rhodes M., 2006. Graduate Skills and Recruitment in the City. City of London y Financial Services Skills Council.

Domina, T., 2007. Higher Education Policy as Secondary School Reform: Texas Public High Schools after Hopwood. Educational Evaluation and Policy Analysis. 29(3), 200-217.

Elloumi, F., 2004. Value chain analysis: A strategic approach to online learning. En: Anderson, T. y Elloumi, F. (Eds). Theory and practice of online learning. Athabasca University, Canada, 61-92.

Feller, A., Shunk, D. y Callarman, T., 2006. Value chains versus supply chains. BP trends, March Edition.

Fernie, J. and Clive, R., 1995. Supply chain management in the national health service. The International Journal of Logistics Management, 6 (2), 83-92.

Froeschle, R., 2010. Labor Supply/Demand Analysis: Approaches and Concerns. TWC Labor Market and Career Information (LMCI).

Gabriel, E., 2006. Value Chain for Services. A new dimension of "Porter's Value Chain". IMS International Journal, 1-30.

Habib, M. y Jungthirapanich, C., 2008a. An integrated framework for research and education supply chain for the universities. Proceedings of the 4th IEEE International Conference on Management of Innovation and Technology. IEEE Computer Society, Piscataway, USA, 1027-32. 
Habib, M. y Jungthirapanich, C., 2008b. Integrated Educational Supply Chain Management (IESCM) for the Universities. Sixth AIMS International Conference on Management, India.

Habib, M. y Jungthirapanich, C., 2009a. A Research Model of Integrated Educational Supply Chain for the Universities. International Conference on Technology and Business Management, Dubai, March.

Habib, M. y Jungthirapanich, C., 2009b. Research Framework of Education Supply Chain, Research Supply Chain and Educational Management for the Universities. International Journal of the Computer, the Internet and Management (IJCIM), Thailand, 17 (SP1), 24, 1-8.

Habib, M. y Jungthirapanich, C., 2009c. Integrated educational management for the universities. The Journal of China-USA Business Review, David Publishing Company, USA, 8 (8), 25-38.

Habib, M. y Jungthirapanich, C., 2009d. Research Framework of Educational Supply Chain Management for the Universities. IEEE International Conference on Engineering Management and Service Sciences EMS. China, September.

Habib, M. y Jungthirapanich, C., 2010. International Supply Chain Management: Integrated Educational Supply Chain Management (IESCM) Model for the Universities. En: Gupta, S.L. International Retailing: Text and Readings. Excel Books, 1-18.

Habib, M. y Jungthirapanich, C., 2010b. An Empirical Study of Educational Supply Chain Management for the Universities. INFORMS International Conference on Industrial Engineering and Operations Management. Bangladesh, January,

Habib, M. y Jungthirapanich, C., 2010c. An Empirical Research of Integrated Educational Management for the Universities. The 2nd IEEE International Conference on Information Management and Engineering. China, April,

Habib, M. y Jungthirapanich, C., 2010d. An Empirical Research of Educational Supply Chain for the Universities. The 5th IEEE International Conference on Management of Innovation and Technology. Singapore.

Habib, M., 2010. An Empirical Study of Tertiary Educational Supply Chain Management. International Conference on Knowledge globalization, 2010”. Bangladesh.

Habib, M., 2010a. An Empirical Research of ITESCM (Integrated Tertiary Educational Supply Chain Management) Model. Management and Services, Sciyo.com. October, ISSN 978-953-7619-X-X. 
Hammer, A., 2006. Enabling Successful Supply Chain Management Coordination, Collaboration, and Integration for Competitive Advantage. Doctoral Dissertation, University of Mannheim, Alemania.

Hansen, H.J., 2008. The value chain and life-long learning. Working paper. VIA University College, Horsens, Denmark.

Harris, R., 1998. Decision Making Techniques., http://www.virtualsalt.com/ crebook6.htm, (Septiembre, 2011).

Harvey, L., 2002. Enhancing employability, recognising diversity, Making Links between higher education and the world of work, Universities UK.

Lau, A.K.W., 2007. Educational supply chain management: a case study. Emerald Group Publishing Limited, ISSN 1074-8121, 15 (1), 15-27

Makkar, U., Gabriel, E., Tripathi, S.K., 2008. Value chain for higher education sector-case studies of India and Tanzania. Journal of Services Research, Special Issue, 184-201.

Mason, G., Williams, G., Cranmer, S. y Guile, D., 2003. How Much Does Higher Education Enhance the Employability of Graduates? HEFCE, RD13_03.

Mora, M., Hassin, K., Pullin, A. y Muegge, S., 2008. Open educational resources and the evolving value chain of education in developing countries. IEEE International Symposium on Technology and Society (ISTAS).

Nixon, M., 2001. Innovations in logistic technology: generating top-line value and bottom-line ROI. World Trade, 14 (6), 62-4.

O’Brien, E.M. y Kenneth, R., 1996. Educational supply chain: a tool for strategic planning in tertiary education? Marketing Intelligence \& Planning, 14 (2), 33-40.

OECD, 2005. Changing supply and demand for S\&T professionals in a globalised economy.

Pathak, V. y Pathak, K., 2010. Reconfiguring the higher education value chain. Management in Education, 24(4), 166-71.

Porter, M., 1985. Competitive Advantage, Creating and Sustaining Superior Performance. The Free Press, New York

Roldán, D., Hervás, A., 2007. A Framework for On-Line Learning Business Models Analysis in Higher Education Institutions. IADIS International Conference, Lisboa.

Sampson, S.E., 2000. Customer-supplier duality and bidirectional supply chains in service organization. International Journal of Service Industry Management, 11 (4), 348-64 
Sengupta, S. y Turnbull, J., 1996. Seamless optimization of the entire supply chain. IIE Solutions, 28 (10), 28-33.

Sison, R. y Pablo, Z.C., 2000. Value Chain Framework and Support System for Higher Education. Proceedings of the Philippine Computing Science Congress (PCSC).

Stevenson, W.J., 2002. Operations Management 7th ed. McGraw-Hill/Irwin, NY

Stylianou, A.C., y Turner, W., 1999. Electronic Academia: A Competitive Analysis based on the Value Chain Model. Proceedings of the International Decision Sciences Institute. Athens, Greece, July.

Tyran, C.K. y Coakley, J.R., 2000. The E-Commerce Course Project: Creating Educational Linkages with the Value Chain. Journal of Informatics Education and Research, 2 (2), 59-70.

Van der Merwe, A y Cronje, J., 2004. The Educational Value Chain as a modelling tool in re-engineering efforts. En: Waldron, J. (Ed.). Proceedings of the 3rd International Symposium on Information and Communication. Las Vegas, Nevada, 122-27.

Walters, D. y Rainbird, M., 2004. The demand chain as an integral component of the value Chain. The Journal of Consumer Marketing, 21, 465.

E\&A

Ward, R. y Pierce, D., 2003. Employability and students' educational experiences before entering higher education. Enhancing Student Employability Co-ordination Team \& LTSN Generic Centre.

Watton, P. y Truscott, J.B. (2006). Enhancing student employability \& entrepreneurship through the environmental and natural sciences. Centre for Excellence in Teaching and Learning (CETL) in Experiential Learning (EL). 